\title{
Optimising the Fronthaul Connectivity and Backhaul Utilization via DWDM using BTS Hotel Concept
}

\author{
Mohd Yusdee bin Yusoff, Mohammad Yazid bin Mohammad, Suzi Seroja Sarnin, Nani Fadzlina \\ Naim
}

\begin{abstract}
The research proposal will be focusing on the benefit for Mobile Network Operators (MNOs) in the backhaul connectivity. In normal situation, each of Base Transceiver Station (BTS) will need to carry their backhaul using any Transmission link either Microwave, Fiber or VSAT to be connected to the Core Network to establish a complete cycle of connectivity in Mobile Network. This backhaul will carry an individual link of each BTS (end site) site that is depends on number of hub before it reach to the Core Network. By introducing the Backhaul Grooming using BTS Hotel Concept will benefit MNOs in cost reduction on numbers of hub/link towards reaching the Core Network. Besides, the solution is economical where the BTS site footprint and Power Consumption will be reduced. The solution will be using the DWDM concept via Fiber optics network where multiple BTS sites will be connected and groom in One location so called BTS Hotel. In this BTS Hotel, the Baseband Unit (BBU) of NodeB/eNodeB will be placed in rack while the Remote Radio Unit (RRU) will remain at End site/station. The connection between BBU and RRUs is called Fronthaul and the connection will be grooming using Dense Wave-length Division Multiplexing (DWDM) connectivity.Using this solution, multiple End Sites will be connected to one single point called BTS Hotel and from this Hotel/room the Backhaul connection to the Core Network or Evolved Packet Core (of each Telco) will be only ONE Single connection instead off multiple link-up from each End site where this solution is very economical as compared to conventional method that currently practice in the industry. The fronthaul connectivity is monitored by the MNOs monitoring center as the RRUs and BBU monitored via NMS (Network Monitoring System) while the backhaul connectivity and utilization will be then monitored via the backhaul usage and can be upgraded accordingly when there is a need subject to site utilization.
\end{abstract}

\section{INTRODUCTION}

$\mathrm{E}$ conomic is a major concern by Telcommunication industry in Malaysia or even worldwide. There are many methods or solutions towards cost saving in the mobile network environment.

This manuscript is submitted on $26^{\text {th }}$ November 2019 and accepted on $16^{\text {th }}$ April 2020. Mohd Yusdee bin Yusoff, Mohammad Yazid bin Mohammad, Suzi Seroja Sarnin and Nani Fadzlina Naim are with the Faculty of Electrical Engineering, Universiti Teknologi MARA, 40450 Shah Alam, Selangor (email: myy1971@gmail.com)

1985-5389/C 2021 The Authors. Published by UiTM Press. This is an open access article under the CC BY-NC-ND license (http://creativecommons.org/ licenses/by-nc-nd/4.0/).
Besides the radio parts i.e. NodeB/eNodeB, the bandwidth of backhaul connectivity to connect the end site to the Core Network is an another solution to be considered. A new concept so called BTS Hotel is introduced in mobile network were the solution will benefit the Mobile Network Operators (MNOs) in lower down the operating cost where mainly focus in bandwith utilization.

The BTS Hotel concept will be using the fiber optic as a medium for the Fronthaul and Backhaul connectivity. In studies this connectivity is also knowns as Cloud Radio Access Network [1]. This solution is preferably build in the metropolitan or green field area. The End site will be connected via fiber optic where only RRU will be installed at End Site location. In the other hand, the BBU will be then installed at one single location called BTS Hotel room and the room is completely installed with Rectifier and Battery Back-Up unit. These End site and BTS Hotel room will be connected using fiber via DWDM concept.

In normal situation the NodeB/eNodeB that consist of RRUs and BBU will be installed at the End site while the backhaul connectivity is via individual link either via fiber or microwave to be connected to Core Network. The backhaul connectivity is mandatory for each site and it is costly.

By introducing this BTS Hotel concept, the backhaul of multiple End sites will be connected to one location (BTS Hotel room/location) and to be carried to the Core Network/Evolved Packet Core (EPC) via one single link instead of one link each. This solution is cost effective as the bandwidth of multiple End Sites can be optimized accordingly. Besides sharing the backhaul, the fronthaul connection between RRUs of one End Site station to its BBU are also been grooming where a pair of fiber optics cables using DWDM technology used to connect this NodeB/eNodeB. This fronthaul connectivity is an another value add in cost saving effenciency in the mobile network. Thus, deployment of this proposal is cost effective in term of 
fronthaul, backhaul as well as saving on space or footprint [2] and tideness of site.

\section{BACKGROUND STUDY}

The growth of the sharing based has been identified as another factor to be considered in network deployment. Not limited to the Mobile or radio part, but it also consider at the fronthaul and backhaul area.

In the industry the Evolved Node B (eNodeB) [3] in LTE technology have 2-types of configuration known as Distributed Type and Macro Type. The Distributed Type is the combination of RRU [4] and BBU [5] while the Macro Type is the equipment that is combination of RF Module and Baseband Module in one single box.

The study will focus on the Distributed type of the eNodeB grooming the multiple RRU Mobile Operators (MNOs) at End Sites to be connected to their BBU using DWDM technology[4]. DWDM is the fiber optic devices that used to combined multiple optical transmitters over one single core of fiber optics cable.

In ideal situation, a complete configuration of one NodeB[3] or eNodeB, the connection between RRUs and their BBU is via fiber called Common Public Radio Interface (CPRI) [6] cables and this connection require Giga bit/s speed and this is called Fronthaul connectivity. The CPRI is a fiber optic cable with multiprotocol transmitting signal used in Distributed Type of eNodeB equipment. Table 1 shows the bit rates required to connect RRU and BBU. Information from this table will reflects to the type of DWDM interface to be used to connect between DWDM equipment and the RRUs. The fronthaul connectivity also limited to fiber optic medium where the copper, microwave media are not feasible to be used.

TABLE 1

REFERENCE OF BIT RATES SPEED BASED ON TYPE OF INTERFACE

\begin{tabular}{|c|c|c|c|}
\hline Usage & Types of Interface & Quality & Rate \\
\hline \multirow{4}{*}{ Iub Interface } & $\mathrm{E} 1$ & 8 & $2 \mathrm{Mbps}$ \\
\cline { 2 - 4 } & $\mathrm{T} 1$ & 8 & $1.5 \mathrm{Mbps}$ \\
\cline { 2 - 4 } & $\mathrm{E} 3$ & 2 & $34.4 \mathrm{Mbps}$ \\
\cline { 2 - 4 } & $\mathrm{T} 3$ & 2 & $44.7 \mathrm{Mbps}$ \\
\cline { 2 - 4 } & STM-1 & 2 & $155 \mathrm{Mbps}$ \\
\hline \hline \multirow{2}{*}{$\begin{array}{c}\text { Interface between BBU } \\
\text { and RRU }\end{array}$} & $\begin{array}{c}\text { CPRI Ethernet } \\
\text { (Optical port or } \\
\text { electrical port) }\end{array}$ & 3 & $100 \mathrm{Mbps}$ \\
\hline \hline \multirow{2}{*}{\begin{tabular}{c} 
Interface between BBUs \\
\cline { 2 - 4 }
\end{tabular}} & Ela & 1 & $3 \times 2.5 \mathrm{Gbps}$ \\
\cline { 2 - 4 } & Elb & 1 & $3 \times 2.5 \mathrm{Gbps}$ \\
\hline
\end{tabular}

Source from Huawei Technologies Inc.

The studies of the CPRI line rates of the fronthaul evolutuion [7] shows that the line rates in between BBU and RRUs is very high and it is not recommended to apply the CPRI connectivity via leased line concept where it will incurred high cost in Operation Expenditure (OPEX). Hence the application over the DWDM is the most efficient and cost effective in the proposal. Table 2 shows the comparison of the existing CPRI line rates used in the industry based on frequency spectrum allocated by the regulatory. Even with the minimum spectrum of $10 \mathrm{MHz}$, the line rate required is at $614.4 \mathrm{Mbps}$ and it is mandatory that fronthaul to be connected not via other medium except fiber optic. The incremental of line rate is proportionate with the speed of the downlink (DL) of mobile user. Nowadays the implementation of latest technology of Multiple Input Multiple Output (MIMO) [8] by the MNO will be another challenge in the industry. The allocated spectrum of radio frequencies offered to $\mathrm{MNO}$ due to cater more users and offering faster in speed. The spectrum is related to bandwith where the larger the bandwidth the more amount of data can be transmitted in a fixed amount of time where bandwidth is expressed in bits per second. The projection for this MIMO and massive MIMO application the approximate line rate is shown in Table 2 and it is very huge and the use of fiber is mandatory.

TABLE 2

EXISTING CPRI/ORI INTERFACE SPECIFICATIONS AGAINST PROJECTED BITRATES FOR RRU LINKS.

\begin{tabular}{|c|c|c|c|}
\hline \multicolumn{2}{|c|}{ Current CPRI/ORI interfaces } & \multicolumn{2}{|c|}{ Projected requirements } \\
\hline Line rate & Example Use & Possible uses & Approx. line rate \\
\hline $614.4 \mathrm{Mb} / \mathrm{s}$ & $10 \mathrm{MHz}$ LTE channel & $\begin{array}{c}100 \mathrm{MHz}, 8 \text { antennas } \\
\text { (sectors/MIMO/CoMP) }\end{array}$ & $28 \mathrm{~Gb} / \mathrm{s}$ \\
\hline $4.9152 \mathrm{~Gb} / \mathrm{s}$ & $\begin{array}{c}8 \times 10 \mathrm{MHz} \\
\text { (multiple antennas) } \\
\end{array}$ & $\begin{array}{c}500 \mathrm{MHz}, 8 \text { antennas } \\
\text { (sectors/MIMO/CoMP) }\end{array}$ & $141 \mathrm{~Gb} / \mathrm{s}$ \\
\hline $10.1376 \mathrm{~Gb} / \mathrm{s}$ & $\begin{array}{c}10 \times 20 \mathrm{MHz} \\
\text { (multiple antennas) }\end{array}$ & $\begin{array}{l}500 \mathrm{MHz}, 16 \mathrm{x} 8 \\
\text { massive MIMO }\end{array}$ & $2.25 \mathrm{~Tb} / \mathrm{s}$ \\
\hline
\end{tabular}

The research on reducing of the fronthaul has been studied by introducing the Split-PHY Processing (SPP) architecture [9]. In order to reduce the bandwidth of the fronthaul connectivity, changes of functional split point in between BBU and RRU need to be done at the layer of LTE MAC and PHY layer, this is known as MAC-PHY split [10]. Figure 1 shows the simulation done by the researcher, where the normal C-RAN or BBU-RRU line rates indicated as Mobile Fronthaul (MFH) is maintained as reference at $7.86 \mathrm{Gbps}$. Then, by using the SPP the concept, the MFH line rates is reduced accordingly and propotionate to the Modulation Coding Scheme (MCS) of QPSK (MCS index: 5,10) at 95\%, 16 QAM (MCS index: 16, $19)$ at $90 \%$ and 64 QAM (MCS index: 25,28$)$ at $85 \%$.

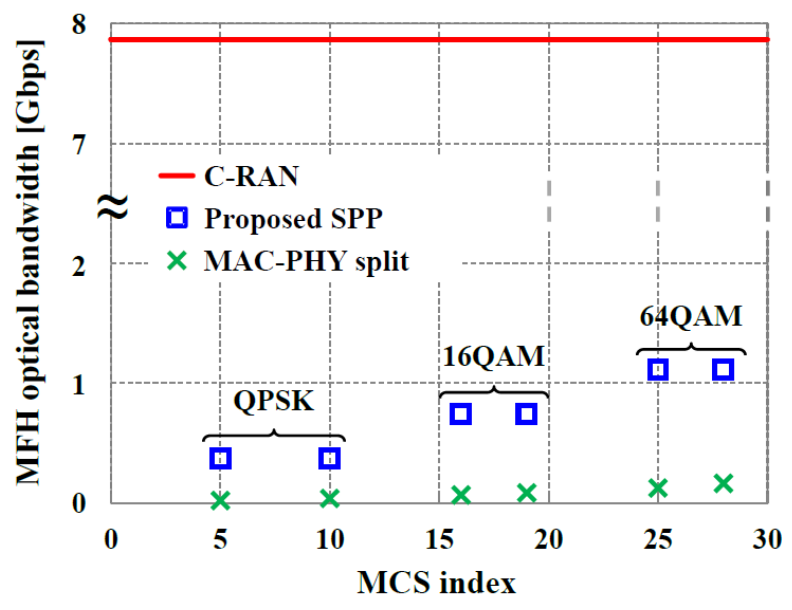

Fig. 1: Mobile Fronthaul (MFH) Optical bandwidth against Modulation Coding System (MCS) Index 
In Figure 2 shows the comparison of the required Signal-toInterference-plus-Noise Ratio (SINR) against MCS index between C-RAN or BBU-RRU rates and SPP. The difference for MCS of QPSK and 16 QAM is $3 \mathrm{~dB}$ while it improved to $2 \mathrm{~dB}$ difference for 64 QAM MCS. These results confirmed that the proposed SPP concept may achieve wireless performance of C-RAN or BBU-RRU line rates/bandwidth connectivity.

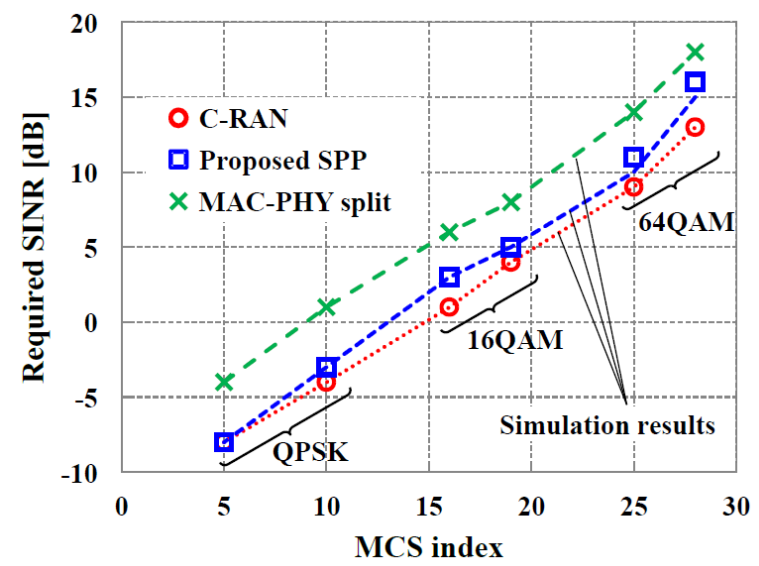

Fig. 2: SINR(dB) against Modulation Coding Scheme (MCS)

\section{CONVENTIONAL SOLUTION BY MNOS}

In normal practice, MNOs setup their NodeB/eNodeB via standard configuration where the RRUs and BBU via CPRI. The standard configuration for one single site will be 3-units of RRU per technology with 1-unit BBU [11].

In standard configuration network shown in Figure 3, in ideal situation 1-technology will occupied 3-RRUs to be connected with 1-BBU. In the case of 3-technologies, $2 \mathrm{G}, 3 \mathrm{G}$ and $4 \mathrm{G}$, total of RRUs will be 9-RRUs required to be installed at End Site and each site require 1-backhaul connection to be carried to MNOs core network or known as Evolved Packet Core (EPC) [12]. In the case of multiple End Sites to be commissioned in one area, the numbers of backhaul will proportionate with the numbers of End Sites. This will cause of MNOs to spend extra cost to commission the End Sites accordingly.

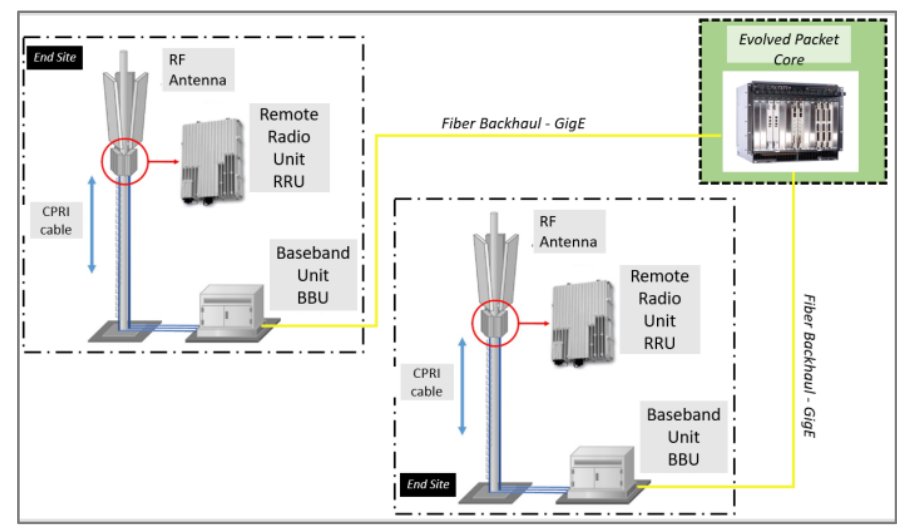

Fig. 3: Base Station Network Connectivity - Standard Configuration

\section{NEW PROPOSED SOLUTION}

Since the cost of network setup is crucial for MNOs nowadays, a BTS Hotel [13] concept is proposed to be used to minimize the Capital Expenditure (CAPEX) and OPEX. The use of fiber optics as medium to connect the End Station and BTS Hotel room is a mandatory as it require high speed bit rate, as mentioned in Table 1 and Table 2.

The Fronthaul using DWDM will be used to connect the RRUs to BBU via 1-pair of fiber for each End Site. While the grooming of multiple BBUs at the BTS Hotel location will only require one single connection to bring back the traffic to the core network of each MNOs. The Fronthaul will connect the RRUs to the BBU via DWDM and the increase of RRUs are manageable by this DWDM module.

The backhaul utilization will be then optimized based on utilization and manageable in the case of low or high traffic utilization via back end control center.

The network diagram of this Fronthaul-Backhaul connectivity is shown as per Figure 4. It shows that the BBUs are grooms together in one single location (Hub Station/BTS Hotel) while the RRUs are located at the End Site to serve MS users. The range of the End Site towards BTS Hotel room is subject to the RRU capability sets by the manufacturer.

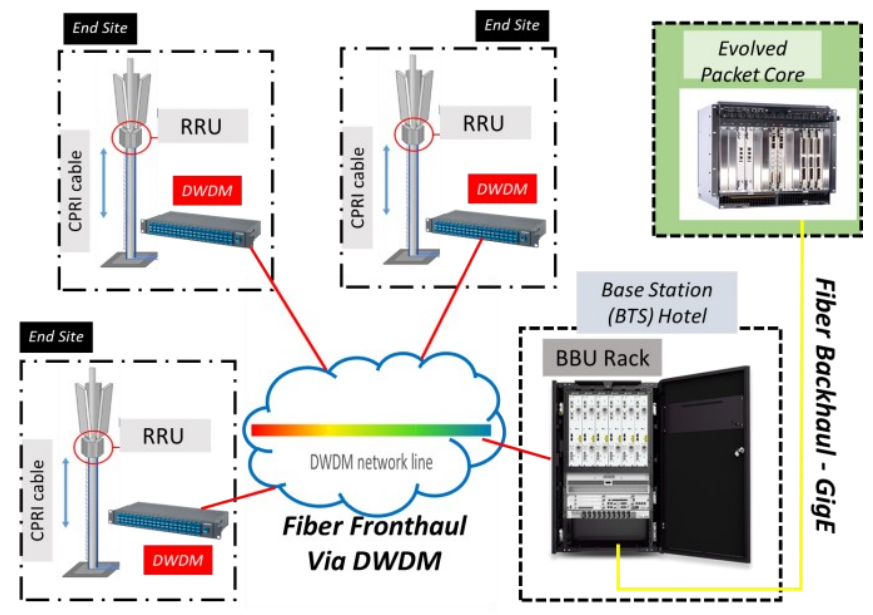

Fig. 4:Fronthaul-Backhaul connectivity

At the End Station, all RRUs installed at the pole and CPRI cable connection connected to the DWDM equipment. This connection applied to all End Station where only 2-cores of fiber are required to bring back the signal (from RRUs) to the Hotel where BBU(s) are located.

These multiple BBUs will then groom together at the BTS Hotel and only One single backhaul connection (Gigabit Ethernet) is required to connect them to their own EPC of each MNOs.

The backhaul utilization will be then monitored by system called Network Traffic Analyzer [14], where the bandwidth or traffic utilization been monitored periodically as per shown in Figure 5. The traffic monitoring is important for MNOs as it works as the reference for them to manage their traffic of their End Sites towards Core Network. Once the traffic reached the target KPI, say $70 \%$ utilization, MNOs may consider increasing their Backhaul connectivity accordingly as to serve their MS users at their End Site. With this controlled mechanism, MNOs will benefits on their backhaul utilization management. In the case of MNOs need an additional or increase of bandwidth due 
to forecast demand, the system can be configured the request accordingly. By using the study case of Radial Traffic Analyzer [14], the tool is able to monitor and visualize the utilization of the user(s) activity and behaviour through their computer or tools.

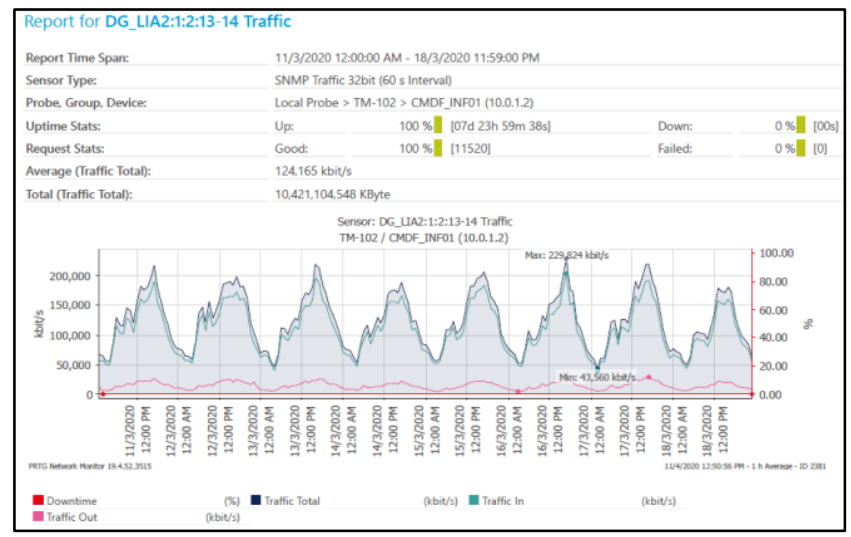

Fig. 5: Network Traffic Monitoring by PRTG Software for single site

The efficiency and effectiveness of the proposed studies in the industries is shown in Table 3 by comparing the cost incurred in between the conventional method against the BTS Hotel concept as discussed in the proposal studies.

TABLE 3

COST COMPARISON FOR MNO BETWEEN BTS HOTEL CONCEPT AGAINST CONVENTIONAL METHOD

\begin{tabular}{|c|c|c|c|c|c|c|c|c|}
\hline Site \# & $\begin{array}{c}\text { Distance } \\
\text { to } \\
\text { Nearest } \\
\text { Fiber } \\
\text { Node } \\
(\mathrm{km})\end{array}$ & $\begin{array}{c}\text { Fiber cost per } \\
\text { km } \\
\text { (RM) }\end{array}$ & $\begin{array}{l}\text { Total Cost } \\
\text { (lay own fiber) } \\
\text { (RM) }\end{array}$ & $\begin{array}{c}\text { BW } \\
\text { Leasing } \\
\text { per } \\
\text { 1Mbps } \\
\text { (RM) }\end{array}$ & \begin{tabular}{|c|} 
BW \\
Required \\
per Site \\
(Mbps)
\end{tabular} & $\begin{array}{c}\text { Total Cost BW } \\
\text { Leased Line } \\
\text { (BWLL) } \\
\text { per month } \\
\text { (RM) }\end{array}$ & $\begin{array}{l}\text { BWLL } \\
\text { 1-year } \\
\text { (RM) }\end{array}$ & $\begin{array}{c}\text { ROI } \\
\text { Return of } \\
\text { Investment } \\
\text { Period } \\
\text { (Comparing } \\
\text { BWLL vs Own } \\
\text { Build Fiber } \\
\text { Facilities) } \\
\text { (Year) } \\
\end{array}$ \\
\hline Site A & 5 & RM160,000 & RM800,000 & RM20 & 250 & RM5,000 & RM60,000 & 13 \\
\hline Site B & 5 & RM160,000 & RM800,000 & RM20 & 250 & RM5,000 & RM60,000 & 13 \\
\hline Site C & 5 & RM160,000 & RM800,000 & RM20 & 250 & RM5,000 & RM60,000 & 13 \\
\hline Site D & 5 & RM160,000 & RM800,000 & RM20 & 250 & RM5,000 & RM60,000 & 13 \\
\hline Site E & 5 & RM160,000 & RM800,000 & RM20 & 250 & RM5,000 & RM60,000 & 13 \\
\hline \multicolumn{2}{|c|}{${ }^{*}$ assumption of $5 \mathrm{~K}$} & $\begin{array}{l}\text { Total Cost for } \\
\text { 5-End Sites }\end{array}$ & RM4,000,000 & & & $\begin{array}{c}\text { Total cost } \\
\text { BWLL for 1- } \\
\text { year for } \\
\text { 5-sites } \\
\end{array}$ & RM300,000 & $\begin{array}{l}13 \text { years } \\
\text { ROI }\end{array}$ \\
\hline
\end{tabular}

Table 3 shows the cost compilation and comparison with assumption of the End Site is at average of $5 \mathrm{~km}$ distance to the nearest Point of Present (PoP) or known as fiber node that could connect the End Site to their EPC. The far the distance will impact more cost on fiber deployment. The PoP normally have an established connection to the EPC and it can be aggregated according to traffic requirement. The Bandwidth (BW) leasing is the backhaul connectivity to carry the BW required per end site to the EPC and the charging is normally based on rates and distance. As for this case study, the BW leasing per 1Mbyte is a reference with $250 \mathrm{Mbps}$ required for each End Site. By comparing the Fiber deployment against BW leasing via BTS Hotel concept, the MNO will benefit a lot where the total cost where the Return of Investment (ROI) will be returned after 13years of operation. So, it can be concluded that the proposed BTS Hotel concept via DWDM is very cost effective for MNO to operates their network to serve the subscribers. The mobile backhaul studies between Fiber optic against Microwave [15] has been highlighted where the fiber network is critical in supporting the technology growth in bandwidth demand.

Besides this proposed solution, other approach and mechanism towards the End Station can also be considered. Among them is Common Antenna Sharing (CAS) [16] of RF Antenna where MNOs may share the same antenna to be more economical and cost effective. This CAS will be then deployed with multi-ports as well as multi bands of frequency range from low-band to high-band, subject to individual MNOs strategy. By imposing the CAS concept, the End Station can be designed with aesthetic value that blend with the surrounding area. The footprint of the End Site will be more tidy with less numbers of equipment cabinate as well as common sharing of power supply rectifier unit. The footprint of End Site will be smaller as compared to conventional method that currently imposed by MNOs nowadays. Figure 6 is a sample of End Site where it is built with CAS with RRUs installed at site (shown the tideness of the site) and the BBU is located in the BTS Hotel room.

As of overall benefits of this proposal, MNO will benefit on CAPEX, the $3^{\text {rd }}$ party (Network Facilities Provider - NFP) who operates and maintaining the BTS Nework setting will generates their revenue from site rental and BW leasing and the Fiber provider who layed their fiber infrastructure will laverage their infra usage not only to residents or commercial customer but they benefits from the fiber core leasing to MNO via BTS Hotel facilities.

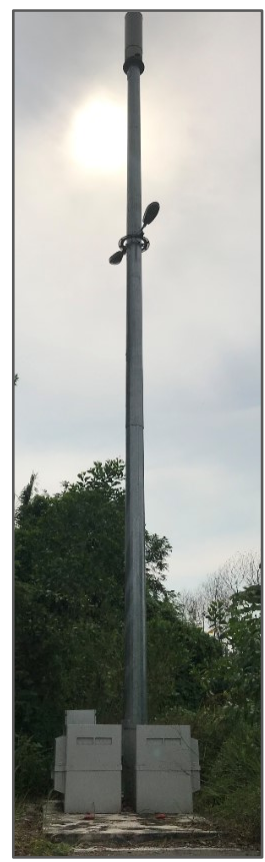

Fig. 6: End Site of BTS Hotel - Common Antenna Sharing (CAS), Power Sharing (Rectifier) and using fronthaul connectivity

The simulation of this studies is describe in network line diagram shown in Figure 7 where the case study is done with comissioning of 6-End Sites and a BTS Hotel using the DWDM concept. The PTN is the Private Transmission Network that used to be a switch for Backhaul connectivity to connect all 
BBUs in the BTS Hotel room to the EPC of the MNO and the interconnection is done at the Point of Interconnection (POI).

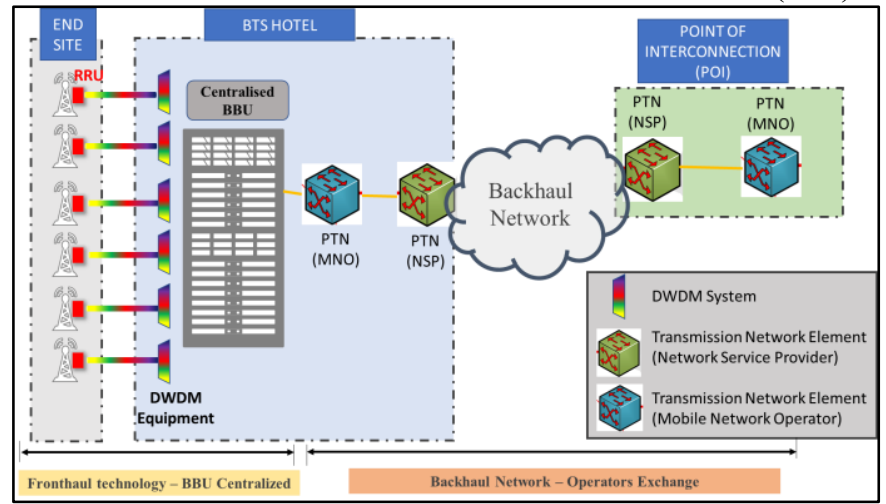

Fig. 7 : BTS Hotel Network Configuration of fronthaul and Backhaul connectivity

All the 6-End Sites only have RRUs installed at end station and connected via DWDM Network known as a fronthaul network while the BBUs are installed in a subrack in the BTS Hotel Room. The simultaion is applied in the stadium and all 6End Sites are installed with 2-technolgies (LTE2600 and UMTS2100). In conventional method, these 6-End Sites will need $6 \times 250 \mathrm{Mbps}$ capacity with total of $1.5 \mathrm{Gbps}$ backhaul connection to EPC. But in this scenario, the BTS Hotel concept where all 6-End Sites are connected via PTN is connected equipped with capacity of $600 \mathrm{Mbps}$ only. The trraffic simulation is then monitored during the event and the results is shown in Figure 8.

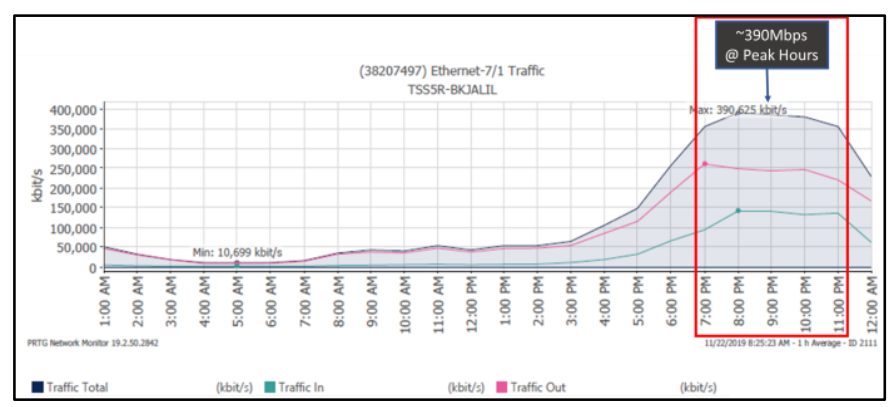

Fig. 8: Traffic of 6-End Sites during major event

By using this DWDM facilities, where allocated bandwidth connected between BTS Hotel to EPC is at $600 \mathrm{Mbps}$ and the utilization is $65 \%$ (390Mbps). By comparing to standard configuration, it is almost half of the total backhaul capacity sets for 6-End Sites of conventional configuration in used.

Figure 9 shows the details traffic seggregation capping at $390 \mathrm{Mbps}$ during Peak Hours which is from $7 \mathrm{pm}$ until $11 \mathrm{pm}$.

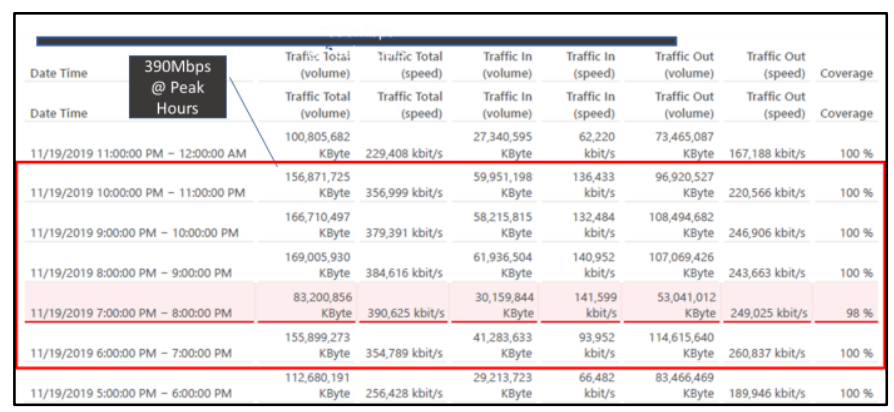

Fig. 9: Traffic statistic during Peak Hours
As a results, the saving on bandwidth is very cost effective by using the BTS Hotel network concept where the use of DWDM capable of doing bandwidth sharing for all sites. Besides, the capacity setting is also flexible and scalable where MNO may allocate more bandwidth to site(s) that based on traffic congestion.

\section{CONCLUSION}

The research proposal is recommended to be applied in the Telecommunication industry as it reflects to the economical in Capital Expenditure (CAPEX) in backhaul as well as smaller space and tideness as well as value in site aesthetical.

\section{ACKNOWLEDGMENT}

The authors wish to thank members of Faculty of Electrical Engineering, Universiti Teknologi MARA Shah Alam, Malaysia for the guidance and support during this project. This research was supported by "Fundamental Research Grants Scheme", file No. 600-IRMI/FRGS 5/3 (099/2017).

\section{REFERENCES}

[1] M. A. Rt, "Smart Backhauling and Fronthauling of Flexibly Centralized Radio Access Networks," no. October, pp. 105-111, 2015.

[2] F. Tonini, M. Fiorani, M. Furdek, L. Wosinska, C. Raffaelli, and P. Monti, "Minimum cost deployment of radio and transport resources in centralized radio architectures," 2016 Int. Conf. Comput. Netw. Commun. ICNC 2016, 2016.

[3] J. A. De Vriendt and A. Lucent, "Mobile Network Evolution: A Revolution on the Move," no. May 2002, 2013.

[4] R. Antil and S. Beniwal, "An Overview of DWDM Technology \& Network," vol. 1, no. 11, pp. 43-46, 2012.

[5] C. Yu, "Distributed Base Station System and Method for Networking Thereof and Base Band Unit," vol. 2, no. 12, 2011.

[6] P. Examiner, R. Ngo, A. Examiner, and G. Mui, "CPRI-Based Multiprotocol Signal Transmission Method And Apparatus in Distributed Base Station System," vol. 2, no. 12, 2010.

[7] J. Nathan, "Fronthaul Evolution: from CPRI to Ethernet," 2015.

[8] Y. E. G. Larsson and B. Labs, "Massive MIMO for Next Generation Wireless Systems," pp. 1-20, 2014.

[9] K. Miyamoto, S. Kuwano, J. Terada, and A. Otaka, "Performance evaluation of mobile fronthaul optical bandwidth reduction and wireless transmission in Split-PHY Processing architecture," pp. 24, 2016.

[10] A. Maeder, M. Lalam, A. De Domenico, and E. Pateromichelakis, "Towards a Flexible Functional Split for Cloud-RAN Networks," no. July 2015, 2014.

[11] C. Liu, S. Member, L. Zhang, S. Member, M. Zhu, and S. Member, "A Novel Multi-Service Small-Cell Cloud Radio Access Network for Mobile Backhaul and Computing Based on Radio-Over-Fiber Technologies," vol. 31, no. 17, pp. 2869-2875, 2013.

[12] W. Hahn, "Flat 3GPP Evolved Packet Core Improvement for multiple network connections," pp. 3-6.

[13] A. Dato and V. Radmilovic, "(12) Patent Application Publication (10) Pub. No.: US 2010/0301212 A1,” vol. 1, no. 19, 2010.

[14] D. A. Keim, F. Mansmann, and T. Schreck, "Monitoring Network Traffic with Radial Traffic Analyzer," vol. 2006, no. Vast 2006, 2008

[15] C. Study, A. Various, and B. Technology, "Mobile Backhaul : Fiber vs . Microwave," no. October, 2009.

[16] P. Yelle, M. A. Us, A. Adc, and W. Solutions, "Multi-Protocol Distributed Antenna System for Multiple Service Provider-Multiple Air Interface Co-Located Base Stations," vol. 2, no. 12, 2010. 